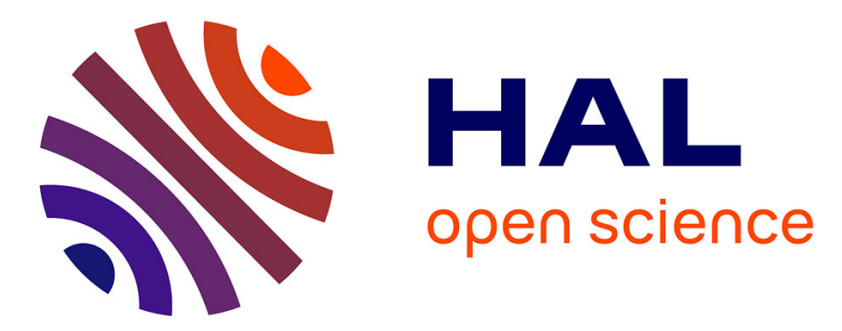

\title{
Performance Evaluation of JT CoMP Approach: Tractable Model Using Spatial Fluid Modeling
}

Lynda Zitoune, Stefan Cerovic, Danilo Cerovic, Véronique Vèque, Jean-Marc

Kelif

\section{- To cite this version:}

Lynda Zitoune, Stefan Cerovic, Danilo Cerovic, Véronique Vèque, Jean-Marc Kelif. Performance Evaluation of JT CoMP Approach: Tractable Model Using Spatial Fluid Modeling. 2016 IFIP Networking Conference (IFIP Networking), May 2016, Vienne, Austria. 10.1109/ifipnetworking.2016.7497216. hal-01411038

\author{
HAL Id: hal-01411038 \\ https://hal.science/hal-01411038
}

Submitted on 22 Sep 2020

HAL is a multi-disciplinary open access archive for the deposit and dissemination of scientific research documents, whether they are published or not. The documents may come from teaching and research institutions in France or abroad, or from public or private research centers.
L'archive ouverte pluridisciplinaire HAL, est destinée au dépôt et à la diffusion de documents scientifiques de niveau recherche, publiés ou non, émanant des établissements d'enseignement et de recherche français ou étrangers, des laboratoires publics ou privés. 


\title{
Performance Evaluation of JT CoMP Approach: Tractable Model using Spatial Fluid Modeling
}

\author{
Lynda Zitoune $^{1,2}$, Stefan Cerovic ${ }^{1}$, Danilo Cerovic $^{1}$, Véronique Vèque $^{1}$, Jean-Marc Kelif ${ }^{3}$ \\ ${ }^{1}$ Signals and Systems Laboratory, CentraleSupelec \\ 3, Rue Joliot-Curie, 91192 Gif sur Yvette, France. \\ \{lynda.zitoune, veronique.veque\} @l2s. centralesupelec.fr \\ \{stefan.cerovic, danilo.cerovic\}@supelec.fr \\ ${ }^{2}$ Department of Systems Engineering, ESIEE-Paris \\ 2, boulevard Blaise Pascal, 93162 Noisy le Grand, France. \\ Iynda.zitounedesiee.fr \\ ${ }^{3}$ Orange-Labs \\ 38-40, rue du Général Leclerc, 92794 Issy Les Moulineaux, France. \\ jeanmarc.kelifeorange.com
}

\begin{abstract}
Résumé-Inter-cell interference is a major issue in OFDMA networks, due to the increasing density of Low Power Nodes (LPN) used to offload the macro base stations. Coordination between these nodes also called Coordination MultiPoint (CoMP), is identified as a promising solution to improve the signal quality and the achievable throughput while ensuring spectral efficiency over the network. Joint Transmission (JT) mode of CoMP consists to jointly transmit the useful signal from more than one BS, typically the best serving one, and one or several other base stations. In dense networks, the performance evaluation and the analysis of JT CoMP approach become a hard task which needs lot of time and huge resources to conduct simulations. In this paper, we present a new mathematical framework based on spatial fluid modeling which reduces the analysis complexity and provides a macroscopic evaluation of the performance, quite faithful to those obtained using Monte Carlo simulations. The key idea is to consider a continuum of nodes rather than a fixed finite number, and to derive the mean impact of a density of nodes in a certain region of the network. The closed-form formulas of the downlink interference factor are defined for three scenarios depending on the number of coordinated nodes. Then, they are used to evaluate the signal quality improvement, particularly at the cell edge.
\end{abstract}

Keywords-Interference mitigation, joint transmission, coordination multipoint, OFDMA network, spatial fluid modeling.

\section{INTRODUCTION}

Today wireless networks become more and more dense in terms of the number of base stations (BSs), access points (APs) and mobile systems (MSs). Increasing the density of BSs and APs is one of solutions to face the exponential growth of wireless data traffic, to improve the critical application QoS (Quality of Service), and to fulfill the increasing demand of users [1]-[4].

Dense cellular networks are of two types [1]-[3]. In urban areas, operators often resort to additional cellular infrastructure to fulfill the users demand and to ensure application QoS, making the already existing cellular network more dense [5]. Furthermore, heterogenous networks are also considered as dense where macro BSs are used together with several Low ISBN 978-3-901882-83-8 (c) 2016 IFIP
Power Nodes (LPN) such as, pico or femto BSs, to form a k-tier network [6], [7]. Small cells are used to offload macro BSs and hence enhance the coverage and the capacity of the overall network and improve the throughput of the end users [8]. However, due to the limited radio resources, the interference is unavoidable and leads to neutralize the benefits of densification by decreasing the quality of the signal received by the MSs mainly at the cell edge [9]-[12], and consequently reducing the network throughput.

Many solutions are used to resolve the interference impact between adjacent cells, also called inter-cell interference [9], [13]-[15]. For example ICIC technique (Inter-Cell Interference Coordination) (release 8 of LTE) and its enhanced version (eICIC) (release 10) are based on some resource scheduling, frequency reuse or power control to reduce the interference at the cell edge in homogeneous and heterogeneous networks respectively. Nevertheless, these approaches present some limitations in case of saturation, i.e. when the terminal number grows, and induce a serious issue on throughput reduction. Recently, Coordinated MultiPoint approach (CoMP), under standardization by 3GPP for LTE-Advanced technology (release 11), is considered as a promising solution to provide high spectral efficiency where destructive interference is turned to constructive one. Some projects have dealt with the practical CoMP schemes in both downlink and uplink communications, and assessed their performance using simulations and field trials to demonstrate the maturity of such approaches as in [9], [13]. Joint Transmission (JT) and Coordinated Beamforming/Scheduling $(\mathrm{CB} / \mathrm{CS})$ are two ways to mitigate interference using coordination [9], [12], [16]. In the former, the coordination is performed based on data sharing/exchanging between coordinated BSs. In the last one, coordination is based on the channel state information exchange in order to select the appropriate beams avoiding interference.

In this paper, we focus on the Joint Transmission method of CoMP when applied in OFDMA network where no intra-cell interference exists. Radio resources in OFDMA are parallel and orthogonal. Technically, a User Equipment (UE) receives multiple signals of the same information from several eNodeBs (denoted eNBs in this paper) together with its serving eNB. 
All these eNBs form a coordinated set. As a consequence, JT coordination improves the cell throughput since all resource blocks are used to transmit only useful information. It enhances the signal quality, mainly at the cell edge, and extends the BS coverage [17]. A new control plan is defined to integrate the coordination, also called CloudRAN, to design the coordinated set, and to deal with issues related to synchronization and resource scheduling on the backhaul network between involved eNBs. Some results related to these issues can be found in [9], [13], [18].

To model the operation of JT CoMP, we develop a fluid modeling framework to evaluate its performance on the downlink, in terms of interference factor decrease and its impact on the signal quality received at the UE. The key idea we consider here, is that a continuum number of eNBs, rather than a fixed finite one, is spatially distributed in the network. We derive a tractable model of the downlink interference factor [19] when JT CoMP is used in three different scenarios. We use the underlying model to compute the mean of interference for different UEs placed randomly in the network and also to evaluate the SINR, the main gain metric of JT CoMP. This spatial fluid model, provides a coarse-grained characterization of the network, by considering eNBs density rather than an exact distance that separates each eNB from each UE. Unlike works based on stochastic geometry modeling, which give the average of the performance (like SINR, outage/coverage and throughput) in a typical position, at a given cell [20]. Moreover, stochastic geometry models are most often not tractable, when others point processes rather than PPP (Poisson Point Process) are used to describe the node positions.

The paper contributions are as follow :

- We provide a mathematical model of JT CoMP using a spatial fluid framework, which is tractable making the evaluation of the signal quality easier using simple expression, whatever the location of a UE.

- We investigate the gain of the JT CoMP approach using the obtained model, by considering different number of eNBs in the coordinated set and environment parameters.

- We prove the effectiveness of the spatial modeling as a mathematical framework for dealing with the interference and as a performance evaluation tool even when the eNBs density decreases due to the coordination. We compare the obtained results to those of Monte Carlo simulations of an equivalent hexagonal grid model.

The resulting model of JT CoMP is a powerful tool to investigate the impact of the coordination on the whole network, and not only on a given cell, since the coordination induces more than one cell and enables UEs in neighboring cells to benefit from coordination. So, the model can be easily used by the network operator as a dimensioning/planning tool for coordination between the BSs. We point out that the computational complexity of the model is out of the paper scope, as well as the comparison to the spatial Poisson process. In the latter case, some results can be found in [21].

The paper is organized as follows. In section II we present some related work on JT CoMP modeling using mainly spatial point processes. Next, we introduce a background on the fluid modeling paradigm and the interference factor in section III. Afterwards, the system model of JT CoMP is explained and analytical expressions are detailed for three scenarios depending on the size of the coordinated set in section IV. In section $\mathrm{V}$, we present the numerical results of the underlying analytical expressions and discuss the accuracy of the model toward Monte Carlo simulations of an equivalent hexagonal one. Finally, conclusions and some perspectives are presented in section VI.

\section{RELATED WORK}

CoMP is a coordination technique which involves multiple nodes or BSs to reduce interference at the cell edge and hence increases the network throughput and the spectral efficiency of the radio channel. The coordination is performed between BSs by exchanging data in Joint Transmission (JT) or channel state information in Coordination Beamforming/Scheduling (CB/CS) [9], [14]. In JT, a UE receives the same data from multiple eNBs in the coordinated set defined beforehand. The eNBs in the coordinated set use the same radio resources and as a consequence improve the received signal quality. Commonly, the coordinated set is formed by neighboring eNBs closer to the UE or which provide strongest signal. Moreover, the number of coordinated eNBs is limited in order to address the technical and practical challenges of the backhaul network [9], [13].

Several works dealt recently with the interference issue in both homogeneous and heterogeneous cellular networks, using different modeling approaches and methodologies, for example : game theory [22], simulation and trials [9], [13], and stochastic geometry [5]-[7]. A concise overview on stochastic geometry can be found in [20], [23]. To be inline with the contributions of our work, we present some works on the modeling and the performance evaluation of CoMP using mainly stochastic geometry. In [18], the authors characterize the SINR distribution when coordination multipoint is used and discuss some practical design problems. The main result of this work is that the SINR is increasingly improved, when the number of BSs increases in a ball with a fixed radius. Therefore the gain of cooperation, in terms of coverage, increases with the path-loss exponent. An evaluation of the coverage probability of an heterogeneous network described by a Poisson Point Process is presented in [24]. Two different connectivity models are considered for coordination, 1) nstrongest BS connectivity model where the coordinated set is composed of BSs which provide strongest signal. 2) nnearest BS connectivity model, where the coordinated set is composed of BSs close in each tier. The analysis shows that the n-strongest model is better than the n-nearest one. In [25], the authors consider CB/CS method of CoMP and show that the performance metrics decrease linearly in case of a non ideal backhaul with a large delay. In [26], it is demonstrated that JT is more powerful than $\mathrm{CB} / \mathrm{CS}$ in terms of performance. The improvements in the network performance are approved in [17] using a realistic urban scenario. Depending on the difference between the received signal strength of the serving cell and the coordinated cells an interference map is constructed. The CoMP gain is then derived using the interference map.

A common remark is that all these studies are carried out considering a Poisson Point Process [11], [20], [23], substantiated by the fact that the nodes location is often random and leads to irregular networks. Tractable models are consequently provided for the SINR distribution, coverage/outage ratio and 
the average rate over the network, where different propagation models (fading/shadowing) are considered. However, when other Point Processes are assumed, mainly regular one [27][29], the performance models like SINR and its derivatives are not analytically tractable due to the non-independent nature of points. In this case, either approximations are used to bound the performance parameters [20], or intensive simulations are conducted to validate the models [30], [31].

Motivated by the spatial fluid modeling developed in [19] and [32], we propose here to use this mathematical framework in order to investigate the benefits of JT CoMP in a dense cellular network, and at the same time, the accuracy of the yielding solutions. To meet this objective, we consider a downlink channel of OFDMA network and derive explicit, numerically tractable integral expressions of the interference factor for three different scenarios of coordination. The interference factor in this case depends on several parameters : position to the serving base station, density of BSs in the vicinity and the path-loss function which is distance-dependent. We compute SINR, the key metric, more precisely the SIR as we neglect the noise effect.

\section{BACKGROUND MATERIALS}

Let us consider a single frequency OFDMA wireless network composed of $B$ base stations (BS) (denoted eNodeB (eNB) in this paper), which covers a urban area. We focus on the downlink. The radio resources of a base station are divided in a number of parallel, orthogonal, non-interfering channels (subcarriers), each one transmitting at power $P$. Therefore, only inter-cell interference is considered. The User Equipments are randomly distributed over the network.

\section{A. Interference Factor}

As defined in [19], the interference factor at the user equipment $u$ is defined as the ratio of total power received from other base stations $p_{e x t, u}$, to the power it receives from its serving BS $\mathrm{b}, p_{i n t, u}$, such as : $f_{u}=\frac{p_{e x t, u}}{p_{i n t, u}}$. Since in OFDMA the sub-carriers are assumed to be orthogonal, therefore intra interference does not exist, so $p_{i n t, u}=P_{b} g_{b, u}$ is the useful power, $P_{b}$ is the power transmitted by the eNB $\mathrm{b}$, and $g_{b, u}$ is the inverse of the path-loss between the serving eNB and the UE $u$. The interference factor can be expressed as : $f_{u}=\frac{1}{P_{b} g_{b, u}} \sum_{j \neq b}^{B} P_{j} g_{j, u}$, where $P_{j}$ denotes the power transmitted by eNB $j$, and $g_{j, u}$ the inverse of the path-loss between the eNB $j$ and the UE $u$. $B$ represents the number of eNBs considered in the cellular radio system.

As a consequence, the quality of the received signal characterized by the Signal to Interference plus Noise Ratio defined as :

$$
S I N R=\frac{P_{b, u}}{P_{e x t, u}+N}
$$

At the UE $u$, the SINR can be expressed as :

$$
S I N R=\frac{1}{f_{u}+\sigma}
$$

Where $N$ is the Gaussian noise and $\sigma=\frac{N}{P_{b, u}}$. Let notice that since all subscribers transmit at the same power $\mathrm{P}$, we can

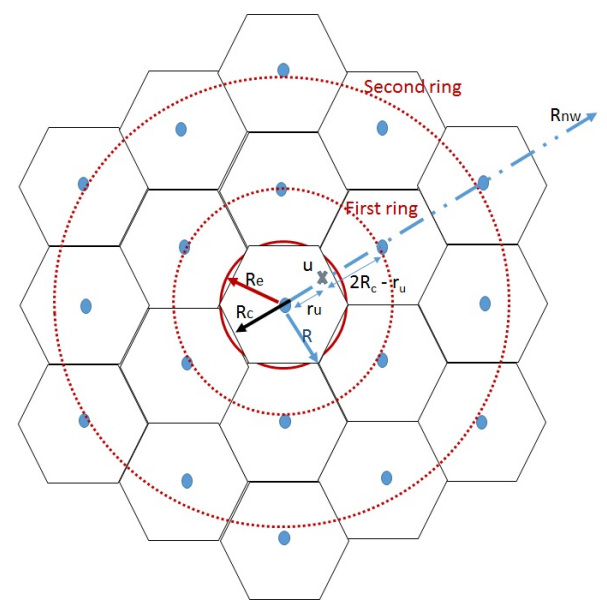

FIGURE 1. The network fluid model and the equivalent hexagonal model.

write for each subscriber :

$$
f_{u}=\frac{1}{g_{b, u}} \sum_{j \neq b}^{B} g_{j, u}
$$

In the case of an homogeneous network, $f_{u}$ depends only on the number of base stations, their positions, the positions of UEs, and their corresponding path-losses [19].

\section{B. Short overview of fluid model}

In fluid network modeling [19], [32], the main assumption is that a fixed finite number of eNBs is replaced by an equivalent continuum of eNBs which are spatially distributed in the network. So basically, the transmitting power of all the eNBs is considered as a continuum field all over the network, and in the case of a uniform eNB distribution and a uniform traffic, the network is considered to be homogeneous. Considering a path-loss model given by [33], $g_{b, u}$ is expressed as $K r^{-\eta}$ where $K$ is a constant, $r$ represents the distance between a UE $u$ and its serving BS, and $\eta>2$ is the pathloss exponent. To reduce the complexity of the model, we neglect the effect of the shadowing and focus our analysis on the other parameters (the shadowing will be considered in a future paper). Therefore, $f_{u}$ also depends on distance $r$. So $f_{u}$ in the equation (2) can now be written as a function of $r$ and not anymore as a function of $u$. A new parameter $\rho_{e N B}$ is introduced which represents the density of BSs. It is constant since the network is assumed to be homogeneous, so that all the eNBs $j$ have the same output power $P_{j}$. As in [19], we will consider a circle shaped network around the cell of interest, where half of the distance to the nearest base station is $R_{c}$, as shown on the Figure 1. We will assume that the whole network radius is $R_{n w}$, and that there are no transmitters at the distance greater than that.

The following distances of interest will be used throughout this paper, and they are represented on the Figure $1: 1) R$ the hexagonal cell radius, 2) $R_{c}$ half of the distance to the nearest eNB, 3) $R_{e}$ the radius of an equivalent disc (i.e. a disc with the same area as the hexagon). 


\section{Network without coordination : fluid model}

As the assumption of a continuum BS is considered in the fluid paradigm, to calculate the amount of external power at certain location in a cell, we can sum up the influence of each small subsurface, $z d z d \theta$, in the area of interest in the network. In other words, the external power received at the UE $u$ located at the distance $r_{u}$ from its serving eNB, can be calculated by the integration of $\rho_{e N B} z d z d \theta P_{b} K z^{-\eta}$ over the ring with inner radius of $2 R_{c}-r_{u}$, and the outer radius of $R_{n w}-r_{u}$, as shown on the Figure 1 (see [19] for more details).

$$
\begin{aligned}
p_{e x t, u} & =\int_{0}^{2 \pi} \int_{2 R_{c}-r_{u}}^{R_{n w}-r_{u}} \rho_{e N B} P_{b} K z^{-\eta} z d z d \theta \\
& =\frac{2 \pi \rho_{e N B} P_{b} K}{\eta-2}\left[\left(2 R_{c}-r_{u}\right)^{2-\eta}-\left(R_{n w}-r_{u}\right)^{2-\eta}\right]
\end{aligned}
$$
as

The useful power received by the UE $u$ can be calculated

$$
p_{b, u}=P_{b} K r_{u}^{-\eta}
$$

because in this case the only serving base station is $b$. So, combining the last two equations, we can calculate the interference factor as :

$$
f_{u}=\frac{2 \pi \rho_{e N B} r_{u}^{\eta}}{\eta-2}\left[\left(2 R_{c}-r_{u}\right)^{2-\eta}-\left(R_{n w}-r_{u}\right)^{2-\eta}\right]
$$

We notice that the interference factor depends only on the distance $r$ from base station $b$, so it can be written as a function of $r$, like :

$$
f(r)=\frac{2 \pi \rho_{e N B} r^{\eta}}{\eta-2}\left[\left(2 R_{c}-r\right)^{2-\eta}-\left(R_{n w}-r\right)^{2-\eta}\right]
$$

Note that in the last expression we assumed that $\rho_{e N B}=$ $\left(3 \sqrt{3} R^{2} / 2\right)^{-1}$, so that, it is constant in every point of the network, because of the assumption that traffic is uniform. We can conclude that the interference factor does not depend on the output power of the eNB (homogeneous network), but only on the density of eNBs $\rho_{e N B}$, the radius of a cell $R_{c}$, the size of the considered network $R_{n w}$, and the path-loss parameter $\eta$, which means it can be easily calculated.

We consider coordination between the serving BS $b$ and eNBs of the first ring, since they are the potential nodes which entail the signal quality received by UEs.

\section{System MOdel of A NeTWORK WITH JT-COMP}

In this part, we propose the application of the fluid model when the cooperation between base stations exists in the network, i.e. when JT CoMP technique is used. The issues related to data sharing and synchronization on the backhaul are not considered here. Let $f_{u}^{\prime}$ denote the interference factor when JT CoMP is used, so that :

$$
f_{u}^{\prime}=\frac{P_{e x t}^{\prime}}{P_{i n t}^{\prime}}
$$

The impact of JT CoMP on the calculation of the interference factor is given through the reduction of the external power $p_{\text {ext }}$ given in (3), by the amount of power $P_{C o M P}$ that UE receives from the eNBs which are in cooperation with its serving eNB $b$. This power amount $P_{C o M P}$ is added to the internal power given in (4).

$$
f_{u}^{\prime}=\frac{P_{e x t}-P_{C o M P}}{P_{b}+P_{C o M P}}
$$

We define a new factor $G_{C o M P}=\frac{P_{C O M P}}{P_{\text {int }}}$ as the gain of JT CoMP using the fluid model. The interference factor $f_{u}^{\prime}$ in this case, depends on the $f_{u}$ without coordination and can be defined as follows :

$$
f_{u}^{\prime}=\frac{f_{u}}{1+G_{C o M P}}-\frac{G_{C o M P}}{1+G_{C o M P}}
$$

As in fluid model there are no exact borders between different cells, in order to express the impact of the coordinated eNBs on the UE $u$, we have to define the area of the cooperation, mainly the integration domain and to sum up the influence of each small subsurface, $z d z d \theta$ over it. We define the boundaries of the first ring as $2 R_{c}-r_{u}$ and $4 R_{c}-r_{u}$ as depicted in Figure 1 for the polar axis, since closest eNBs are at the distance $2 R_{c}$ from serving eNB.

The polar angle depends on the number of eNBs in the coordination set. Since in hexagonal lattice model there are six eNBs neighbors around the serving eNB, we propose to divide the first ring into six equal areas, and suppose that each area has the same impact on the UE because of the assumption of homogeneous network. Therefore, we define the integration boundaries of the polar angle from 0 to $n \frac{\pi}{3}$ (because $2 \pi / 6=\pi / 3$ ), $n$ is the number of coordinated eNBs considered in the first ring. As given in the following formula, the power of coordinated stations $b_{1, \cdots, n}$ is expressed as :

$$
p_{b_{1, \cdots, n}, u}=\int_{0}^{\frac{n \pi}{3}} \int_{2 R_{c}-r_{u}}^{4 R_{c}-r_{u}} \rho_{e N B} P_{b} K z^{-\eta} z d z d \theta
$$

and is equal to :

$p_{b_{1, \ldots, n}, u}=\frac{n \pi}{3} \frac{\rho_{e N B} P_{b} K}{\eta-2}\left[\left(2 R_{c}-r_{u}\right)^{2-\eta}-\left(4 R_{c}-r_{u}\right)^{2-\eta}\right]$

Three different scenarios are considered in the following to evaluate the JT CoMP gain when one, two, three eNBs are in the coordinated set.

\section{A. Scenario $1:$ cooperation with one base station in the first ring}

As the simplest case, cooperation between the serving eNB $b$ and one base station in the first ring, $b_{1}$, is considered. In order to express the impact of the eNB $b_{1}$ on the UE, we must do the integration over the one sixth of the first ring, because there are six base stations in the first ring, assuming that each one of them has the same impact on the UE (Figure 2).

As given in the following formula, the power of the coordinated station $b_{1}$ is :

$$
p_{b_{1}, u}=\frac{\pi}{3} \frac{\rho_{e N B} P_{b} K}{\eta-2}\left[\left(2 R_{c}-r_{u}\right)^{2-\eta}-\left(4 R_{c}-r_{u}\right)^{2-\eta}\right]
$$




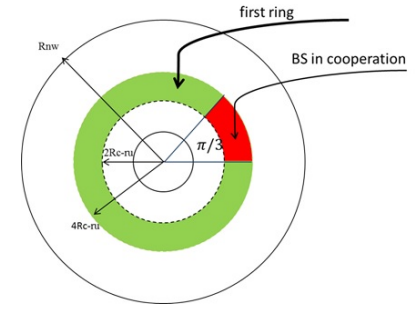

FIGURE 2. Scenario 1 : coordination with one eNB from the $1^{\text {st }}$ ring

So, in this scenario, the total external power is :

$$
\begin{aligned}
p_{e 1} & =2 \pi \frac{\rho_{e N B} P_{b} K}{\eta-2}\left[\left(2 R_{c}-r_{u}\right)^{2-\eta}-\left(R_{n w}-r_{u}\right)^{2-\eta}\right] \\
& -\frac{\pi}{3} \frac{\rho_{e N B} P_{b} K}{\eta-2}\left[\left(2 R_{c}-r_{u}\right)^{2-\eta}-\left(4 R_{c}-r_{u}\right)^{2-\eta}\right]
\end{aligned}
$$

The total internal power is :

$p_{i 1}=P_{b} K r_{u}^{-\eta}+\frac{\pi}{3} \frac{\rho_{e N B} P_{b} K}{\eta-2}\left[\left(2 R_{c}-r_{u}\right)^{2-\eta}-\left(4 R_{c}-r_{u}\right)^{2-\eta}\right]$

We can calculate the interference factor by dividing $p_{e 1}$ defined in (12) by $p_{i 1}$ of equation (13). The improvement compared to the default case in terms of interference factor is obvious since we increase the nominator and decrease the denominator with the same fraction.

\section{B. Scenario 2 : Cooperation with two eNBs in the first ring}

In this case cooperation between serving eNB $b$ and two eNBs $(n=2), b_{1}$ and $b_{2}$, in the first ring is considered,as in Figure 3. So, the reasoning is the same like in the previous case, with the only difference that now two base stations out of six are included in the calculation of the useful power :

$$
p_{b_{1,2}, u}=\int_{0}^{2 \pi / 3} \int_{2 R_{c}-r_{u}}^{4 R_{c}-r_{u}} \rho_{e N B} P_{b} K z^{-\eta} z d z d \theta
$$

This will give us the following expressions for the external power $p_{e 2}$ and the internal power $p_{i 2}$, respectively :

$$
\begin{aligned}
p_{e 2}= & \frac{2 \pi \rho_{e N B} P_{b} K}{\eta-2}\left[\left(2 R_{c}-r_{u}\right)^{2-\eta}-\left(R_{n w}-r_{u}\right)^{2-\eta}\right](15) \\
& -\frac{2 \pi}{3} \frac{\rho_{e N B} P_{b} K}{\eta-2}\left[\left(2 R_{c}-r_{u}\right)^{2-\eta}-\left(4 R_{c}-r_{u}\right)^{2-\eta}\right]
\end{aligned}
$$

$p_{i 2}=P_{b} K r_{u}^{-\eta}+\frac{2 \pi}{3} \frac{\rho_{e N B} P_{b} K}{\eta-2}\left[\left(2 R_{c}-r_{u}\right)^{2-\eta}-\left(4 R_{c}-r_{u}\right)^{2-\eta}\right]$

In this case the interference factor, which we can get by dividing (15) by (16), is even higher than in previous case.

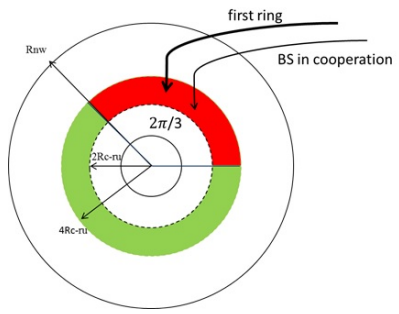

FIgURE 3. Scenario 2 : coordination with two eNBs from the $1^{\text {st }}$ ring

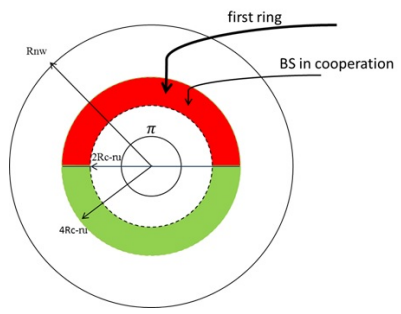

FIGURE 4. Scenario 3 : coordination with three eNBs from $1^{\text {st }}$ ring

\section{Scenario 3 : cooperation with $3 \mathrm{BSs}$ in the first ring}

In this scenario, we consider the cooperation between serving the eNB $b$ and three eNBs in the first ring $\left(b_{1}, b_{2}\right.$ and $b_{3}$ ) as shown in Figure 4 . Now, the external interference is reduced and the internal one is increased by the following amount :

$$
p_{b_{1,2,3}, u}=\int_{0}^{\pi} \int_{2 R_{c}-r_{u}}^{4 R_{c}-r_{u}} \rho_{e N B} P_{b} K z^{-\eta} z d z d \theta
$$

because three base stations out of six make an angle of exactly $\pi$. The external and the internal interference are given with following formulas in this case :

$$
\begin{gathered}
p_{e 3}=\frac{2 \pi \rho_{e N B} P_{b} K}{\eta-2}\left[\left(2 R_{c}-r_{u}\right)^{2-\eta}-\left(R_{n w}-r_{u}\right)^{2-\eta}\right](18) \\
-\pi \frac{\rho_{e N B} P_{b} K}{\eta-2}\left[\left(2 R_{c}-r_{u}\right)^{2-\eta}-\left(4 R_{c}-r_{u}\right)^{2-\eta}\right] \\
p_{i 3}=P_{b} K r_{u}^{-\eta}+\pi \frac{\rho_{e N B} P_{b} K}{\eta-2}\left[\left(2 R_{c}-r_{u}\right)^{2-\eta}-\left(4 R_{c}-r_{u}\right)^{2-\eta}\right]
\end{gathered}
$$

As expected, the interference factor grows even more compared to previous cases.

\section{Simulation AND NUMERICAL RESUlTS}

The objective of this section is twofold. First, we aim to validate the model of JT CoMP for the three scenarios exposed earlier. We compare numerical results of the fluid interference factor to those obtained by Monte Carlo simulations of an equivalent hexagonal model. The second objective is to evaluate the gain of the coordination by computing the SINR and comparing its variation to the case where no coordination is applied between eNBs.

For Monte Carlo simulation, we consider 10 rings of hexagonal cells around a central hexagon of interest such that $R_{n w}=21 R_{c} .50 \mathrm{UEs}$ are generated uniformly in the central 


\begin{tabular}{|c|c|}
\hline Parameters & Values \\
\hline \hline$R$ & $50 \mathrm{~m}$ (femtocell network) \\
\hline$R_{c}$ & $43.30 \mathrm{~m}\left(R_{c}=\frac{R \sqrt{3}}{2}\right)$ \\
\hline$\eta$ & $\in\{2.5,3,3.5,4\}$ \\
\hline$p_{t}$ & $250 \mathrm{~mW}$ \\
\hline Bandwidth & $10 \mathrm{MHz}$ \\
\hline
\end{tabular}

TABle I. Simulation PARAmeters.

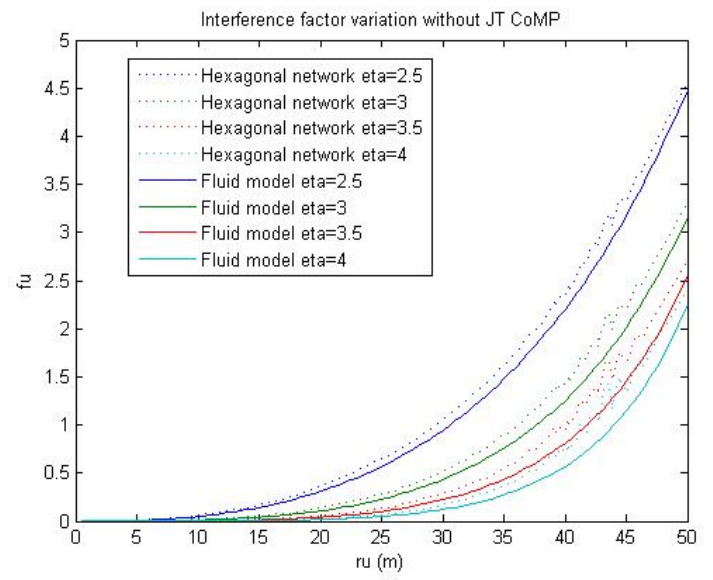

FIGURE 5. Interference factor variation without JT CoMP

hexagon and we suppose that they are attached to the eNB located at the center of this hexagon. We sort all these UEs depending on the distance to their serving eNB, and we average the interference factor for all the UEs at the same distance. To simulate the coordination, the coordinated eNBs are chosen uniformly out of 6 possible eNBs from the first ring depending on each scenario.

The numerical results of fluid modeling are obtained using equations, (6) (without coordination), (12) and (13) for scenario 1, (15) and (16) for scenario 2 and (18) and (19) for scenario 3, over a network of radius $R_{n w}$. The eNBs density is $\rho_{e N B}=\left[\frac{3 \sqrt{3}}{2} \cdot R^{2}\right]^{-1}$ in a central circle of radius $R$ (cf Figure $1, \mathrm{R}$ is drawn in blue arrow). The other simulation parameters common with hexagonal model, are summarized in the table I.

The plot of the interference factor as a function of distance from the serving eNB for the default case, without coordination, is shown in Figure 5. The curves of this figure prove the accuracy of the fluid model towards hexagonal lattice one. These results are pretty similar to those in [19] and [32], and show an obvious result that is, near the serving base station, the interference factor is lower than at the edge. For example for $\eta=2.5$, the interference factor is about 0.25 at $20 \mathrm{~m}$ from the serving eNB, and reaches 2.1 at $20 \mathrm{~m}$ further. $f_{u}$ increases exponentially with the distance whatever the exponent values, and it is inversely proportional to the path-loss exponent, i.e. $f_{u}$ is higher for a lower loss path exponent $\eta=2.5$.

Furthermore, in the default case (Figure 5), we can notice that there is a slight difference between fluid and hexagonal model. This difference is bounded and does not exceed $8 \%$. The difference is related to the circular symmetry around the serving eNB, and the circular shaped form considered in fluid model. So, whatever the position of the UE in the inner circle,

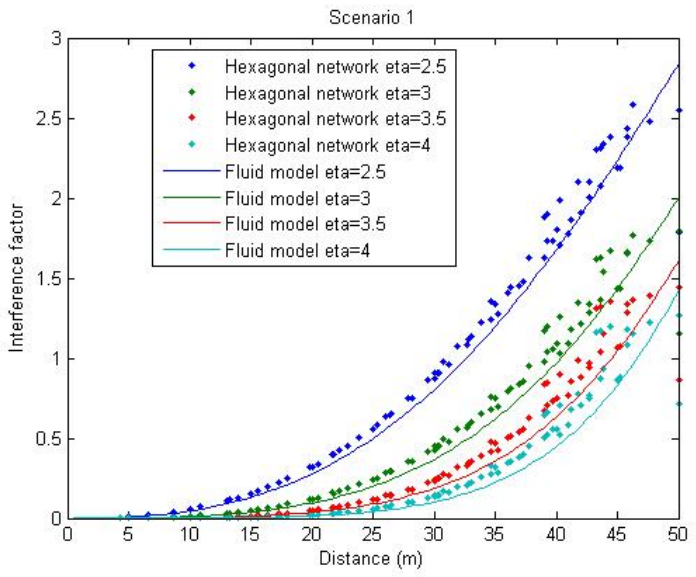

FIGURE 6. Interference factor variation in scenario 1

\begin{tabular}{|c|c|c|c|c|}
\hline & no CoMP & Scenario 1 & Scenario 2 & Scenario 3 \\
\hline \hline$\eta=2.5$ & 4.48 & 2.84 & 2.40 & 1.4 \\
\hline$\eta=3$ & 3.16 & 2.00 & 1.60 & 0.92 \\
\hline$\eta=3.5$ & 2.55 & 1.61 & 1.23 & 0.70 \\
\hline$\eta=4$ & 2.25 & 1.42 & 1.04 & 0.60 \\
\hline
\end{tabular}

TABle II. NumericAl Results of $f_{u}$ AT THE CELL EDGE, $r_{u}=50 \mathrm{M}$.

the average of the external power of all neighboring eNBs is the same. However in the hexagonal model, this assumption is no longer valid.

When eNBs of the first ring are used together with the serving one, the interference factor decreases significantly as shown in Figures 6,10 and 12. In the table II, we give the fluid numerical results of $f_{u}$ at $50 \mathrm{~m}$, at the cell edge for different path-loss exponent. For example for $\eta=2.5$, we observe that the interference factor decreases by a factor of 3.1 when three eNBs are added to the coordinated set.

The plots of SIR (Signal to Interference Ratio) in $\mathrm{dB}$, in Figures 7, 11 and 13 show that fluid model of JT CoMP match very well with Monte Carlo simulations of Hexagonal model. Moreover, the gain of coordination is highlighted in Figures 8 and 9. The gain is increasingly important at the cell edge, and

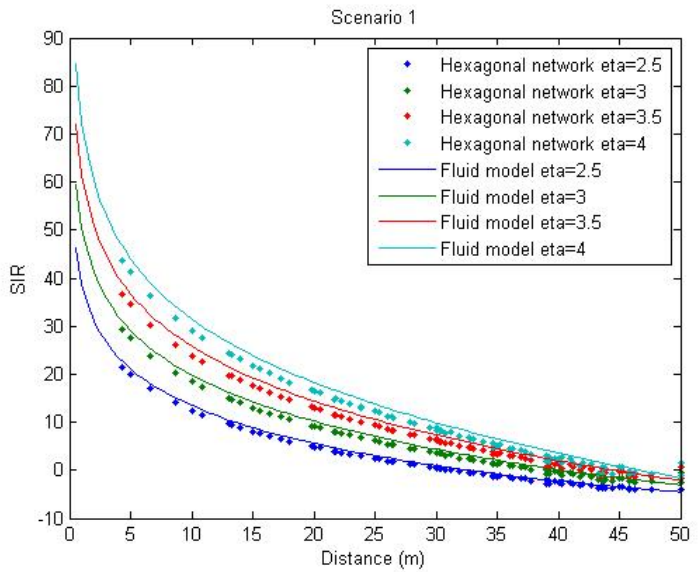

FIGURE 7. SIR variation in case of scenario1 


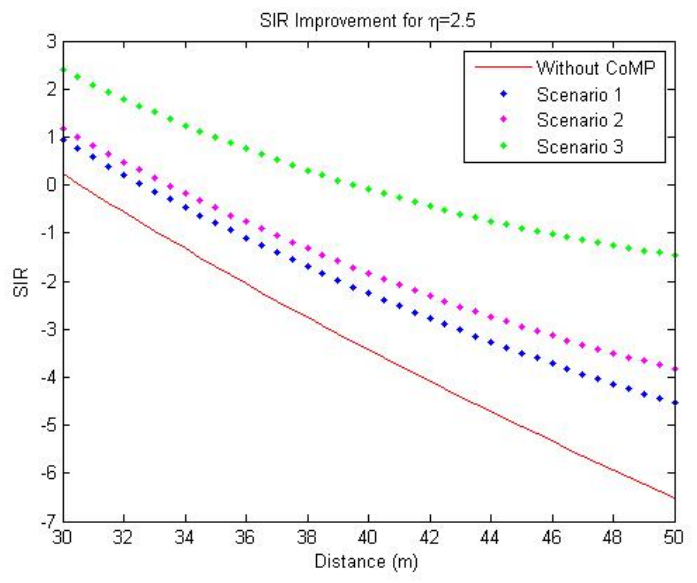

FIGURE 8. SIR improvement, $\eta=2.5$

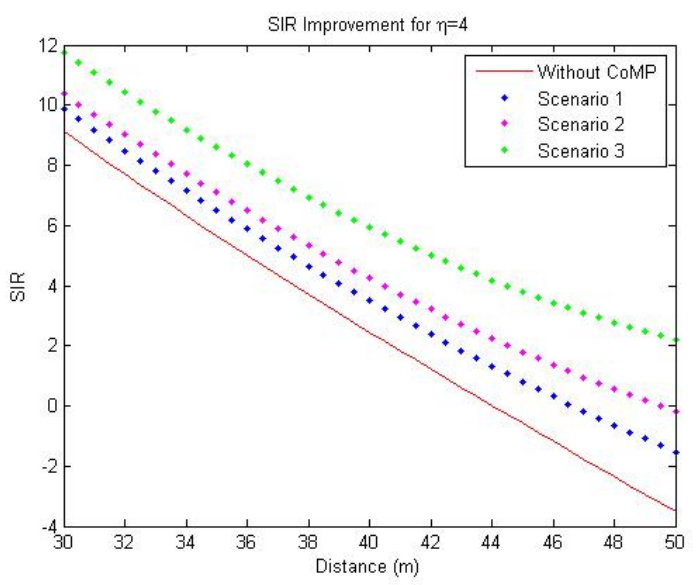

FIGURE 9. SIR improvement, $\eta=4$

we notice that it is quite related to the coordinated BSs number and to the distance from the serving eNB than the path-loss exponent $\eta$. For example at the distance of $46 \mathrm{~m}$ from the serving eNB, the SIR gain is about $4.2 \mathrm{~dB}$ for $\eta=2.5$, and about $4.5 \mathrm{~dB}$ for $\eta=4$. This gain is observed considering the default scenario without CoMP and the scenario3. At the same distance, comparing curves of scenario without CoMP and scenario 2, we observe that the CoMP gain is around $2 \mathrm{~dB}$, in Figures 8 and 9, whatever the value of $\eta$. More generally, the gain of cooperation is two times more important every time a new eNB joins the coordination process, whatever $\eta$.

In the second scenario, 2 eNBs from the first ring cooperate with the serving eNB. They are picked uniformly out of 6 possible eNBs from the first ring around the serving eNB. The plot of the interference factor for this scenario in Figure 10, shows that there is a difference between fluid and hexagonal models, mainly for UEs which are further away from the serving eNB. The difference here is quite higher than that observed for the first scenario in the Figure 6 which is around $12 \%$.

In the third scenario, $3 \mathrm{BSs}$ from the first ring cooperate with the serving BS. They are also picked uniformly out of 6 possible BSs from the first ring around serving BS, as can be

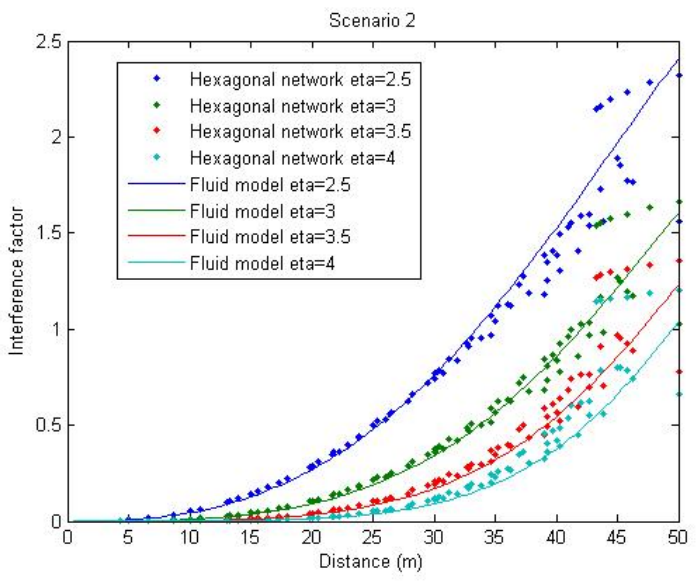

FIGURE 10. Interference factor variation : scenario2

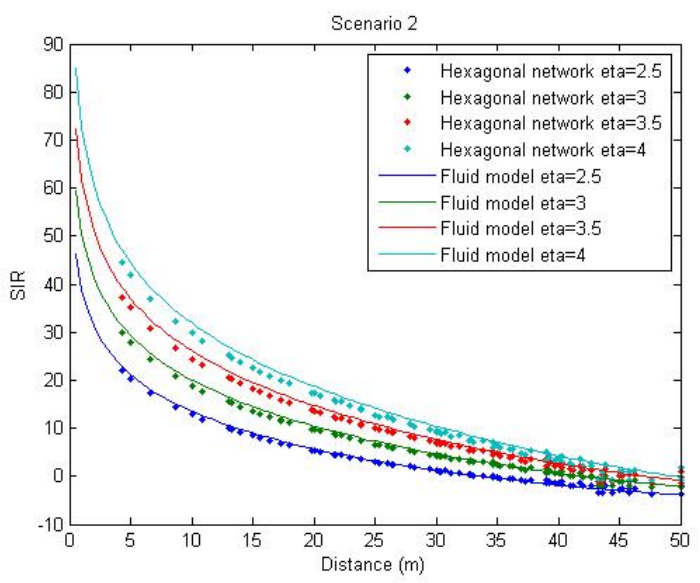

FIGURE 11. SIR variation in case of scenario2

seen in the Figure 4. The plot of the interference factor for this scenario is shown in Figure 12. We notice that there is also a distinction between fluid and hexagonal model for the UEs which are further away from the serving BS. In both scenarios 2 and 3 , the difference between the fluid and hexagonal models is quite higher than the scenario 1 , which is around $12 \%$.

In case of cooperation with one, two or three eNBs of the first ring, the impact of these BSs is subtracted from the external power and added to internal power as shown in the previous section. In the fluid model, the impact of the cooperation is not related to only the eNB or eNBs involved in the cooperation, and the distance separating this or these BSs to the UE. The impact of coordination in fluid modeling is related to a density of eNBs over the sub-area around the UE, which is constant. Moreover, fluid modeling inherently assumes a circular symmetry around the UE in case of coordination. Consequently, whatever the UE position, removing the impact of one, two or three sub-areas from the first ring remains the same. However, in the hexagonal model exact distances between coordinated eNBs and the UE are considered. Furthermore, since coordinated eNBs are picked uniformly out of 6 possible BSs, the coordination gain of far BSs is insignificant compared to the remaining external power. Another point which can explain the difference between the 


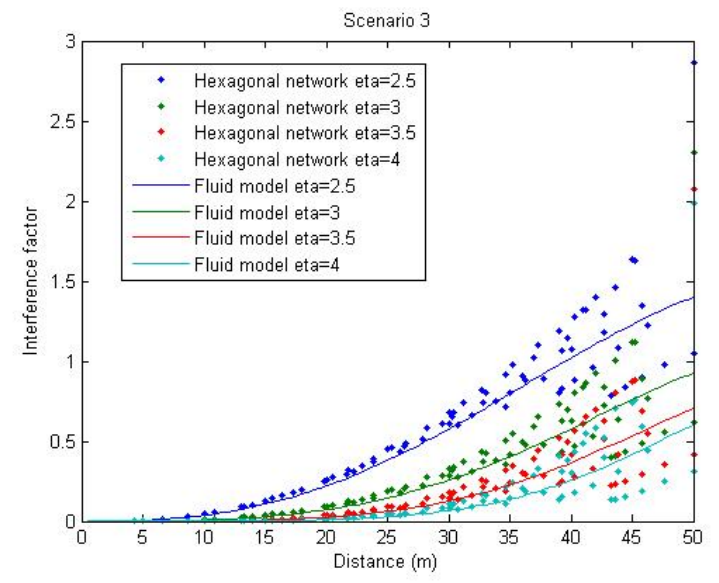

FIGURE 12. Interference factor variation : scenario3

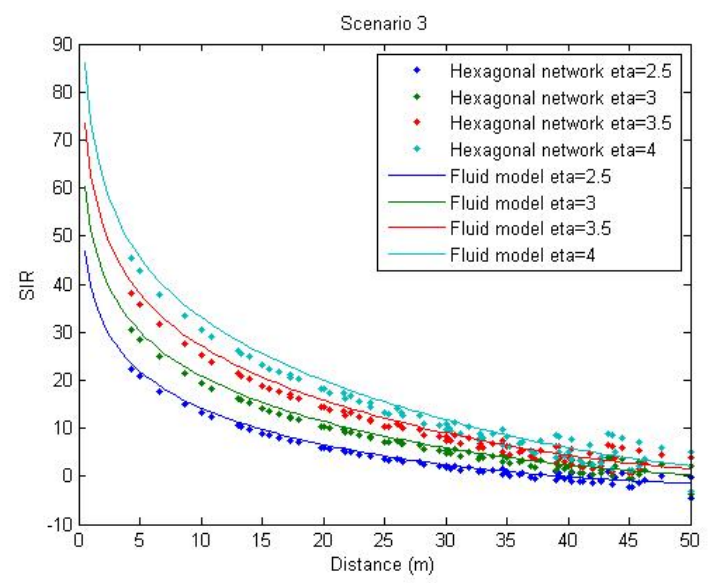

FIGURE 13. SIR variation in case of scenario3

fluid an the hexagonal models, is related to the difference between the area of the hexagon of radius $R$ and the equivalent circle of radius $R e$. So that, the fluid model does not catch the extreme parts of the hexagon.

Indeed, the fluid model gives analytical expressions which do not take into account probability that user will be positioned differently from the CoMP eNB. This is a very interesting feature, since the UE is not compelled to perform measurements to define the best coordinated eNBs, and thus simplifies the multipoint association procedure.

\section{CONCLUSION}

The Joint Transmission of Coordinated MultiPoint approach is used to deal with the inter-cell interference, the main issue emphasized by the increased number of BSs and LPNs (Low Power Nodes). In this paper, we developed a new mathematical framework based on spatial fluid modeling to investigate the gain of this approach on the downlink of an OFDMA network. We considered a continuum number of eNBs spatially distributed in the network rather than a fixed finite number. We derived closed-form formulas of the interference factor for three scenarios depending on the number of coordinated eNBs. These tractable expressions allow us to rapidly compute the SINR of UEs in an OFDMA network.
Numerical results show that the gain of JT coordination is related to the coordinated BSs number and to the distance from the serving eNB than to the path-loss exponent $\eta$. The gain is two times more important every time a new eNB joins the coordination process. Furthermore, the proposed framework reduces considerably the analysis complexity and provides a macroscopic evaluation of the performance, faithful to those obtained using Monte Carlo simulations. Indeed, fluid modeling inherently assumes a circular symmetry around the $\mathrm{UE}$ in case of coordination, and gives analytical expressions which do not take into account probability that user will be positioned differently from CoMP eNB. The last result is very interesting, since the $\mathrm{UE}$ is not compelled to perform measurements to define the best coordinated eNBs, and thus simplifies the multipoint association procedure.

Future work will focus on the characterization of this difference between the fluid and the hexagonal models mainly in the cell edge, by finding an expression for an upper bound of the difference. We aim also to develop expressions of the other metrics like the throughput and the coverage probability, and to investigate the effectiveness of the fluid framework considering the shadowing effect.

\section{ACKNOWLEDGEMENT}

This work is part of the French project LCI4D of the Systematic Cluster, Paris Region Systems \& ICT Cluster (http://www.systematic-paris-region.org/).

\section{RÉFÉRENCES}

[1] C.-X. Wang and al, "Cellular architecture and key technologies for 5G wireless communication networks," IEEE Communications Magazine, vol. 52, no. 2, pp. 122-130, Feb. 2014.

[2] S. Yuas, M. Valkama, and J. Niemela, "Spectral and energy efficiency of ultra-dense networks under different deployement strategies," IEEE Communications Magazine, vol. 53, no. 1, Jan. 2015.

[3] B. Soret, K. Perdersen, N. Jorgensen, and V. Fernandez-Lopez, "Interference coordination for dense wireless networks," IEEE Communications Magazine, vol. 53, no. 1, Jan. 2015.

[4] M. Bennis, M. Simsek, A. Czylwik, W. Saad, S. Valentin, and M. Debbah, "When cellular meets WiFi in wireless small cell networks," Communications Magazine, IEEE, vol. 51, no. 6, 2013.

[5] J. Andrews, F. Baccelli, and R. Ganti, "A tractable approach to coverage and rate in cellular networks," IEEE Transactions on Communications, vol. 59, no. 11, pp. 3122-3134, Oct. 2011.

[6] H. Dhillon, R. Ganti, and J. Andrews, "A tractable framework for coverage and outage in heterogeneous cellular networks," in Information Theory and Applications Workshop (ITA), Feb. 2011, pp. 1-6.

[7] H. Wang, X. Zhou, and M. Reed, "Analytical evaluation of coverageoriented femtocell network deployment," in International Conference on Communications (ICC), June 2013, pp. 5974-5979.

[8] V. Chandrasekhar, J. Andrews, and A. Gatherer, "Femtocell networks : A survey," CoRR, vol. abs/0803.0952, 2008.

[9] R. Irmer, H. Droste, P. Marsch, S. Brueck, H.-P. Mayer, L. Thiele, and V. Jungnickel, "Coordinated multipoint : Concepts, performance, and field trial results," IEEE Communications Magazine, Feb. 2011, iMTAdvanced and Next-Generation Mobile Networks.

[10] A. Daeinabi, K. Sandrasegaran, and X. Zhu, "Survey of intercell interference mitigation techniques in LTE downlink networks," in Australasian Telecommunication Networks and Applications Conference (ATNAC). IEEE, Nov. 2012, pp. 1-6.

[11] H. Dhillon, R. Ganti, F. Baccelli, and J. Andrews, "Modeling and analysis of K-Tier downlink heterogeneous cellular networks," IEEE Journal on Selected Areas in Communications, vol. 30, no. 3, pp. 550560, April 2012 
[12] D. Lee, B. Clerckx, E. Hardouin, D. Mazzarese, S. Nagata, and K. Sayana, "Coordinated multipoint transmission and reception in LTEAdvanced : Deployment scenarios and operational challenges," IEEE Communications Magazine, pp. 148-155, Feb. 2012.

[13] V. Jungnickel and al, "Field trials using coordinated multi-point transmission in the downlink," 21st International Symposium on Personal, Indoor and Mobile Radio Communications Workshops, 2010.

[14] S. Singh, A. Kumar, S. Khurmi, and S. Tanvir, "Coordinated multipoint (CoMP) reception and transmission for LTE-Advanced/4G," International Journal of Computer Science And Technology, vol. 3, June 2012.

[15] A. S. Hamza, S. S. Khalifa, H. S. Hamza, and K. Elsayed, "A survey on inter-cell interference coordination techniques in OFDMA-based cellular networks," IEEE Communications Surveys Tutorials, vol. 15, no. 4, pp. 1642-1670, 2013.

[16] W. Yu, T. Kwon, and C. Shin, "Multicell coordination via joint scheduling, beamforming and power spectrum adaptation," in IEEE INFOCOM 2011, April 2011, pp. 2570-2578.

[17] S. Berger, Z. Lu, R. Irmer, and G. Fettweis, "Modelling the impact of downlink CoMP in a realistic scenario," in IEEE Wireless Communications and Networking Conference (WCNC), April 2013, pp. 3932-3936.

[18] R. Tanbourgi, S. Singh, J. Andrews, and F. Jondral, "A tractable model for non-coherent joint-transmission base station cooperation," pp. 4959 4973, Sept. 2014.

[19] J.-M. Kelif and E. Altman, "Downlink fluid model of CDMA networks," in Proc. of IEEE VTC Spring, May 2005.

[20] R. Heath and M. Kountouris, "Modeling heterogeneous network interference using poisson point processes," in Information Theory and Applications Workshop (ITA), Feb. 2012, pp. 17-22.

[21] J.-M. Kélif, S. Senecal, C. Bridon, and M. Coupechoux, "Quality of service and performance evaluation : A fluid approach for poisson wireless networks," in IEEE International Conference on Network of the Future (NoF), Paris, France, Dec. 2014.

[22] K. Khawam, A. A. S. Lahoud, J. Cohen, and S. Tohme, "Game theoretic framework for power control in intercell interference coordination," in IFIP Networking Conference, June 2014, pp. 1-8.

[23] B. Blaszczyszyn and H. Keeler, "Studying the SINR process of the typical user in poisson networks by using its factorial moment measures," CoRR, vol. abs/1401.4005, 2014.

[24] G. Nigam, P. Minero, and M. Haenggi, "Coordinated multipoint in heterogeneous networks : A stochastic geometry approach," in IEEE GLOBECOM Workshop on Emerging Technologies for LTE-Advanced and Beyond 4G, Atlanta, GA, Dec. 2013.

[25] P. Xia, C.-H. Liu, and J. Andrews, "Downlink coordinated multi-point with overhead modeling in heterogeneous cellular networks," CoRR, vol. abs/1210.5503, 2012.

[26] Y. Yifan, R. Yun, L. MingQi, S. Bin, and S. RongFang, "Achievable rates of coordinated multi-point transmission schemes under imperfect CSI," In Proc. IEEE ICC, pp. 1-6, 2011.

[27] M. Haenggi, "Mean interference in hard-core wireless networks," IEEE Communications Letters, vol. 15, no. 8, pp. 792-794, Aug. 2011.

[28] M. Abdelrahman, T. ElBatt, and A. El-Keyi, "Coverage probability analysis for wireless networks using repulsive point processes," in Personal Indoor and Mobile Radio Communications (PIMRC), Sept 2013, pp. 1002-1007.

[29] I. Nakata and N. Miyoshi, "Spatial stochastic models for analysis of heterogeneous cellular networks with repulsively deployed base stations," Performance Evaluation, vol. 78, pp. 7-17, 2014.

[30] A. Busson, L. Zitoune, V. Vèque, and B. Jabbari, "Outage analysis of integrated mesh LTE femtocell networks," in IEEE Global Communications Conference GLOBECOM, Austin, United States, Dec. 2014.

[31] I. L. Cherif, L. Zitoune, and V. Vèque, "The r-1 square point process : The effect of coordinated multipoint joint transmission," in International Wireless Communications \& Mobile Computing Conference (IWCMC), Dubrovnik, Croatia, Aug. 2015.

[32] J.-M. Kelif, M. Coupechoux, and P. Godlewski, "A fluid model for performance analysis in cellular networks," EURASIP Journal on Wireless Communications and Networking, 2010.
[33] "Guidelines for evaluation of radio interface technologies for IMTadvanced,’ Report ITU-R M.2135-1, Tech. Rep., 2009. 